\title{
A Proof-theoretic Analysis of the Classical Propositional Matrix Method
}

\author{
David Pym ${ }^{1}$, Eike Ritter ${ }^{2}$, and Edmund Robinson ${ }^{3}$ \\ 1 University of Aberdeen, Scotland, UK \\ 2 University of Birmingham, England, UK \\ 3 Queen Mary, University of London, England, UK
}

\begin{abstract}
The matrix method, due to Bibel and Andrews, is a proof procedure designed for automated theorem-proving. We show that underlying this method is a fully structured combinatorial model of conventional classical proof theory.
\end{abstract}

\section{Introduction}

The matrix method is an efficient proof procedure for classical and non-classical logics. The matrix method for classical logic was introduced by Bibel [4] and Andrews [2]. Developments for modal and intuitionistic logics have been given by Wallen [21]. In all cases, the matrix method is defined in terms of truthfunctional semantics. In this paper, we show that, hidden behind this method, is a non-trivial structural semantics for classical proofs with a relatively simple combinatorial structure. Looked at another way, using the denotational semantics for classical proofs recently given by Führmann and Pym [7-9], we give an analysis of the space of proofs characterized by the matrix method.

In recent decades, it has been the received wisdom that the classical sequent calculus has no interesting denotational semantics. Indeed, cartesian closed categories with dualizing maps are equivalent to boolean lattices $[16,12]$. This algebraic observation is, however, too crude to reveal the proof-theoretic structure to be found. To see the point, consider the following argument, usually attributed to Lafont [10], about cut-reduction in the classical sequent calculus:

$$
\begin{array}{cc}
\Phi_{1} & \Phi_{2} \\
\frac{\Gamma{ }^{\prime} \Delta}{\Gamma \vdash \phi, \Delta} W R & \frac{\Gamma \vdash \Delta}{\Gamma, \phi \vdash \Delta} W L \\
\frac{\Gamma, \Gamma \vdash \Delta, \Delta}{\Gamma \vdash \Delta} C L, C R,
\end{array}
$$

in which $\Phi_{1}$ and $\Phi_{2}$ are arbitrary proofs of $\Gamma \vdash \Delta$. Essentially, this proof reduces to either $\Phi_{1}$ or $\Phi_{2}$, the choice being non-deterministic. In a model that interprets cut-reduction as equality, $\Phi_{1}$ and $\Phi_{2}$ acquire the same denotation and the model collapses to a boolean algebra. 
There have been various attempts to avoid the collapse. The classical natural deduction systems $[18,19]$, for example, represent classical proofs as $\lambda$-terms but do not faithfully represent the structure of cut-reduction: different evaluation strategies (e.g., call-by-name, -value) give different choices (resp. $\Phi_{1}, \Phi_{2}$ ) in (1).

Recently, however, Führmann and Pym [7-9] have introduced a quite general class of classical categories that model classical sequent calculus (LK) proofs in poset-enriched linearly distributive categories (with sufficient extra structure to interpret the classical structural rules of contraction and weakening). The posetenrichment models cut-reduction, so that whenever a proof $\Phi$ reduces to a proof $\Psi, \llbracket \Phi \rrbracket \leq \llbracket \Psi \rrbracket$. The semantics illuminates the status of the MIX law in classical logic: the two obvious ways of defining it - as degenerate cuts against either $\perp$ or $T-$ are equated, and the rule can be eliminated. Examples of classical categories are given by Boolean lattices, the two evident versions of the category of relations, Rel, and by Geometry-of-Interaction constructions [8,9]. Classical categories give a sound and complete semantics for classical sequent proofs with cut-reduction. McKinley [17] has shown that the Calculus of Structures yields a model of classical proofs in this sense. Work by Bellin, Hyland, Robinson, and Urban [3] seeks to find a categorical semantics of classical proofs that, in particular, does not validate the $\eta$-laws. Hyland $[12,13]$ provides discussions of a range of issues.

In this paper, we present a simple combinatorial model of classical proofs. We begin, in $\S 2$, with a brief introduction to the semantics of classical proofs in classical categories. In $\S 3$, we describe the classical propositional matrix method $[4,2,21]$. Then, in $\S 4$, we present a direct construction, motivated by the structural organization of the matrix method, of a simple combinatorial model built out of the propositional literals (atoms and their negations), two binary operations - corresponding to conjunction and disjunction - and a relational notion of connection between complementary literals. We provide the requisite soundness and completeness theorems. Finally, in $\S 5$, a technical section about the interpretation of the cut rule, and $\S 6$, we obtain a remarkable result: our combinatorial model is exactly a proof-theoretic characterization of the matrix method of Bibel, Andrews, and Wallen for deciding classical propositional provability [4, $2,21]$, with equality derived from the semantics provided by classical categories.

The combinatorial model we present in this paper is rather extensional. In particular, it provides a direct description of the equivalence classes of proofs in terms of relations between corresponding literals. These relations are sufficient to characterize the equivalence classes of proofs, but at the price of being unable to model the dynamic behaviour of proofs, such as distinguishing which proof rules are applied in which order.

There is also related work on combinatorial models in the relational setting by Došen [6], Hughes [11], and Lamarche and Straßburger [14]. In [15], Lamarche and Straßburger study a detailed relational model of classical proofs very close to ours. The underlying concern of those papers, however, is based on deficiencies in the kind of sequent calculus we have from Gentzen. These deficiencies include the large amount of information in a typical sequent that is simply context for 
the rule being applied, and that needs to be copied. These issues result in a desire to find better representations of proofs that do not suffer from these deficiencies and infelicities, and in that context the models discussed in this paper are too crude.

This paper is not so ambitious. We are not trying to find a definitive geometrical structure for classical proofs. Rather we are producing a new datapoint by observing that a very simple combinatorial structure, which is strongly linked to a distinctly non-structural proof technique, can be seen to carry the algebraic structure that we associate with classical structural proof theory, that that structure is not completely determined (there are choices) and that it is order-enriched in a way that interacts well with cut. The story is as much about the relationship with the matrix method as the model itself.

\section{Classical Categories and the Classical Sequent Calculus}

We give a brief introduction to classical categories [7-9], omitting the details of the commuting diagrams required for coherence. We explain the semantics of the classical sequent calculus in classical categories.

A classical category is a poset-enriched category with extra structure, including two symmetric monoidal products, $\otimes$ and $\oplus$, with units 1 and 0 . Mediating between these functors is a natural transformation $\delta: A \otimes(B \oplus C) \rightarrow(A \otimes B) \oplus C$, making it a symmetric linearly distributive category, subject to some equations $[5,7]$. We also require, for each object $A$, a complement $A^{\perp}$, together with morphisms $A^{\perp} \otimes A \rightarrow 0$ (contradiction) and $1 \rightarrow A \oplus A^{\perp}$ (excluded middle) giving, subject to some coherences [7], a symmetric linearly distributive category with negation (equivalent to a *-autonomous category). The interpretation of conjunction, disjunction, and negation is the evident one.

Given proofs $\Phi$ of $\Gamma \vdash \phi, \Delta$ and $\Phi^{\prime}$ of $\phi, \Gamma^{\prime} \vdash \Delta^{\prime}$, with denotations $\llbracket \Phi \rrbracket$ and $\llbracket \Phi^{\prime} \rrbracket$, respectively, in a classical category $\mathcal{C}$, their cut is denoted very directly by

$$
\llbracket \Gamma \rrbracket \otimes \llbracket \Gamma^{\prime} \rrbracket \stackrel{\llbracket \Phi \rrbracket \otimes / d}{\longrightarrow}(\llbracket \Delta \rrbracket \oplus \llbracket \phi \rrbracket) \otimes \llbracket \Gamma^{\prime} \rrbracket \stackrel{\delta^{\prime}}{\longrightarrow} \llbracket \Delta \rrbracket \oplus\left(\llbracket \phi \rrbracket \otimes \llbracket \Gamma^{\prime} \rrbracket\right) \stackrel{\text { Id } \oplus \llbracket \Phi^{\prime} \rrbracket}{\longrightarrow} \llbracket \Delta \rrbracket \oplus \llbracket \Delta^{\prime} \rrbracket,
$$

where $\delta^{\prime}$ is the evident morphism obtained from $\delta$ and the symmetric monoidal isomorphisms, and Id is identity. Thus, cut is generalized composition.

A classical category also carries the structure necessary to interpret contraction and weakening. For the rules on the right, every object has a symmetric monoid — that is, a multiplication $\nabla_{A}: A \oplus A \rightarrow A$ with unit [ $]_{A}: 0 \rightarrow A$ satisfying associativity and symmetry, and neutrality of []$_{A}-$ such that $\nabla_{A \oplus B}$ and []$_{A}$ are defined pointwise, and []$_{0}=\mathrm{Id}$. Dually, we have symmetric comonoids, given by the evident $\Delta_{A}$ and \langle\rangle$_{A}$, for contraction and weakening on the left.

Definition 1. A classical category is a symmetric linearly distributive category with negation, with a poset-enrichment $\leq$ such that:

1. The symmetric monoidal category $(\mathcal{C}, \oplus, 0)$ has symmetric monoids; 
2. The symmetric monoidal category $(\mathcal{C}, \otimes, 1)$ has symmetric comonoids;

3. The object-indexed families of maps $\Delta_{A}, \nabla_{A},\langle\rangle_{A}$, and [ $]_{A}$ are lax natural transformations:

$$
\begin{array}{cc}
\Delta \circ f \leq(f \otimes f) \circ \Delta & f \circ \nabla \leq \nabla \circ(f \oplus f) \\
\langle\rangle \circ f \leq\langle\rangle & f \circ[] \leq[]
\end{array}
$$

4. The inequalities $\Delta \nabla,\langle\rangle[], \nabla \Delta$, and []\langle\rangle ,
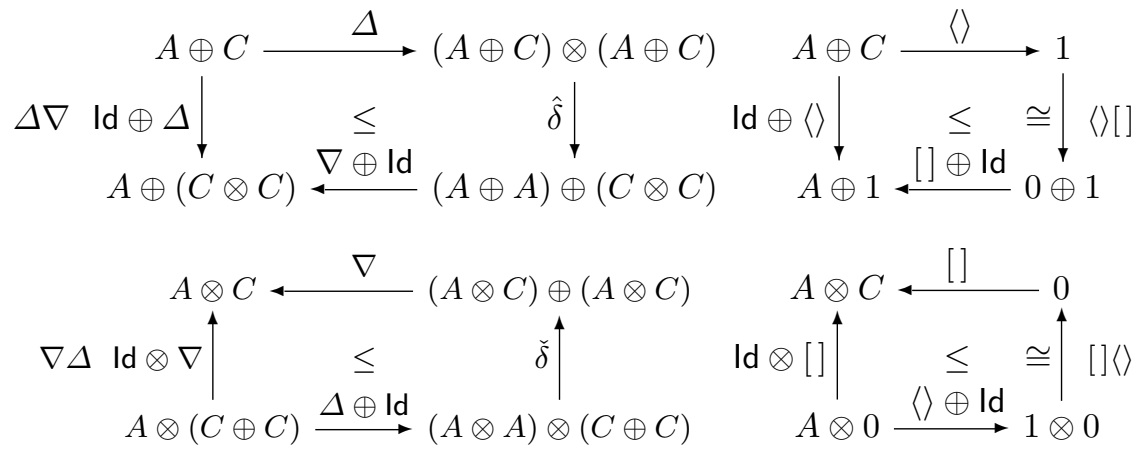

hold, relating $\Delta, \nabla,\langle\rangle$, and [], where $\hat{\delta}$ and $\check{\delta}$ are the evident maps derived from $\delta$ using symmetric monoidal isomorphisms;

5. Composition of morphisms and the functors $\otimes$ and $\oplus$ are monotonic in all arguments.

Example 1. A Boolean lattice is a classical category with meet $\otimes$ and join $\oplus$.

Example 2. Rel $_{\otimes}$ is a classical category with objects sets and morphisms binary relations, in which both $\otimes$ and $\oplus$ given by set-theoretic product. Both 0 and 1 are given by the one-element set, $\{*\}$. Negation is identity on objects. The excluded middle on a set $A$ is the relation $\{(*,(x, x)): x \in A\}$ from $\{*\}$ to $A \times A$. The map $\nabla_{A}$ is $\{((x, x), x): x \in A\}$ and []$_{A}$ is $\{(*, x): x \in A\}$. The order on hom-sets is set-theoretic inclusion.

Example 3. If $\mathcal{C}$ and $\mathcal{C}^{\prime}$ are classical categories, then so are $\mathcal{C}^{o p}$ and $\mathcal{C} \times \mathcal{C}^{\prime}$. In particular, the product of a classical category with non-trivial hom-sets, such as $R e l_{\otimes}$, and one that is non-compact, such as a Boolean lattice (meet as $\otimes$, join as $\oplus$ ), is a non-trivial, non-compact classical category.

A model of the negation-free fragment of classical logic is given by $\mathbf{R e l}_{\oplus}$, in which both disjunction and conjunction are modelled by disjoint union. This is an example of a 'Dummett category' (i.e., a model of the negation-free fragment of classical logic) [9]. In [8,9], Führmann and Pym give a general class of models using a Geometry-of-Interaction construction (e.g., [1]), applied to Dummett categories.

Classical categories provide a sound and complete semantics for classical sequent calculus proofs with cut-elimination via a notion of theory on proofs. The sequent calculus, essentially Gentzen's LK but presented as the calculus for classical propositional linear logic together with the structural rules of contraction and weakening, is given in Table 1 . We assume a language $\mathcal{L}$ of propositional letters. 


$$
\begin{aligned}
& \frac{\Gamma \vdash \phi, \Delta \quad \Gamma^{\prime}, \phi \vdash \Delta^{\prime}}{\Gamma, \phi \vdash \phi, \Delta} \text { Axiom } C u t \\
& \frac{\Gamma, \psi, \phi, \Gamma^{\prime} \vdash \Delta}{\Gamma, \phi, \psi, \Gamma^{\prime} \vdash \Delta} E L \quad \frac{\Gamma \vdash \Delta, \psi, \phi, \Delta^{\prime}}{\Gamma \vdash \Delta, \phi, \psi, \Delta^{\prime}} E R \\
& \frac{\Gamma \vdash \phi, \Delta}{\Gamma, \neg \phi \vdash \Delta} \neg L \quad \frac{\Gamma, \phi \vdash \Delta}{\Gamma \vdash \neg \phi, \Delta} \neg R \\
& \overline{\perp \vdash} \perp L \quad \overline{\vdash \top} T R \\
& \frac{\Gamma, \phi, \psi, \Gamma^{\prime} \vdash \Delta}{\Gamma, \phi \wedge \psi, \Gamma^{\prime} \vdash \Delta} \wedge L \quad \frac{\Gamma \vdash \phi, \Delta \quad \Gamma^{\prime} \vdash \psi, \Delta^{\prime}}{\Gamma, \Gamma^{\prime} \vdash \phi \wedge \psi \vdash \Delta, \Delta^{\prime}} \wedge R \\
& \frac{\Gamma, \phi \vdash \Delta \quad \Gamma^{\prime}, \psi \vdash \Delta^{\prime}}{\Gamma, \Gamma^{\prime}, \phi \vee \psi \vdash \Delta, \Delta^{\prime}} \vee L \quad \frac{\Gamma \vdash \Delta, \phi, \psi, \Delta^{\prime}}{\Gamma \vdash \Delta, \phi \vee \psi, \Delta^{\prime}} \vee R \\
& \frac{\Gamma, \phi, \phi, \Gamma^{\prime} \vdash \Delta}{\Gamma, \phi, \Gamma^{\prime} \vdash \Delta} C L \quad \frac{\Gamma \vdash \Delta, \phi, \phi, \Delta^{\prime}}{\Gamma \vdash \Delta, \phi, \Delta^{\prime}} C R \\
& \frac{\Gamma \vdash \Delta}{\Gamma, \phi \vdash \Delta} W L \quad \frac{\Gamma \vdash \Delta}{\Gamma \vdash \phi, \Delta} W R
\end{aligned}
$$

Table 1. Classical Propositional Sequent Calculus

Definition 2. The sequent theory $\mathcal{T}$ over a collection of atoms $\mathcal{A}$ and a set $\mathcal{I}$ of inequalities $\Phi \preccurlyeq^{\prime} \Psi$ where $\Phi$ and $\Psi$ are proofs of a sequent $\Gamma \vdash \Delta$ over $\mathcal{A}$ is the smallest set of inequalities $\Phi \preccurlyeq \Psi$ between proofs of the same sequent s.t.

1. $\Phi \preccurlyeq \preccurlyeq^{\prime} \Psi$ implies $\Phi \preccurlyeq \Psi$;

2. The relation $\preccurlyeq$ is reflexive, transitive, and compatible, that is, all inference rules are monotonic with respect to $\preccurlyeq$;

3. The relation holds for both directions of the usual cut-reduction rules for logical cuts. It also holds in both directions for a number of coherence rules, including axiom (i.e., $\eta$-) expansions; see [8, 7] for details;

4. The usual rules for eliminating cut against weakening and contraction hold in one direction only, from redex to reduct.

Definition 3. Let $\mathcal{C}$ be a classical category over a collection of atoms $\mathcal{A}$ and let $\mathcal{I}$ be a set of inequalities between sequent proofs. Let $\mathcal{T}$ be the sequent theory over $\mathcal{A}$ and $\mathcal{I}$. An interpretation of $\mathcal{T}$ in $\mathcal{C}$ is a map $\llbracket-\rrbracket$ from formulae to objects and sequent proofs $\Gamma \vdash \Delta$ to morphisms with domain $\llbracket \Gamma \rrbracket$ and co-domain $\llbracket \Delta \rrbracket$ s.t.:

(i) 【-』on formulae respects $\otimes, \oplus$ and negation;

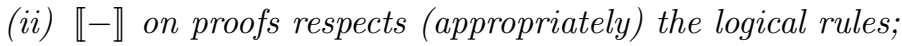

(iii) $\llbracket \Phi \rrbracket \leq \llbracket \Psi \rrbracket$ if $\Phi \preccurlyeq \Psi$ in $\mathcal{T}$. 
Then, we have the following [7]:

Theorem 1 (soundness [7]). Let $\mathcal{C}$ be a classical category over a collection of atoms $\mathcal{A}$ and let $\mathcal{I}$ be a set of inequalities between sequent proofs. Let $\mathcal{T}$ be the sequent theory over $\mathcal{A}$ and $\mathcal{I}$ and let $\llbracket-\rrbracket$ be an interpretation of $\mathcal{T}$ in $\mathcal{C}$. If $\Phi \preccurlyeq \Psi$ in $\mathcal{T}$, then $\llbracket \Phi \rrbracket \leq \llbracket \Psi \rrbracket$ in $\mathcal{C}$.

Via a term model construction (using classical proof nets $[20,7]$ ) we obtain:

Theorem 2 (completeness ([7])). For every set of atoms $\mathcal{A}$ there exists a classical category $\mathcal{C}$ such that for any set of inequalities $\mathcal{I}$ between sequent proofs and all interpretations $\llbracket-\rrbracket$ of the classical sequent theory $\mathcal{T}$ over $\mathcal{A}$ and $\mathcal{I}$ in $\mathcal{C}$ and two sequent proofs $\Phi$ and $\Psi$ of $\Gamma \vdash \Delta$, if $\llbracket \Phi \rrbracket \leq \llbracket \Psi \rrbracket$, then $\Phi \preccurlyeq \Psi$.

Theorem 3 (initiality ([7])). The classical category identified in Theorem 2 is initial in the category of classical categories.

\section{The Matrix Method}

The matrix method, due variously to Bibel [4], Andrews [2], and, for non-classical systems, Wallen [21], is a procedure in automated theorem proving for deciding the provability of propositional formulæ. Here we are concerned with the classical case. We take the exposition as in Bibel [4] as our starting point, but include certain details Bibel left to the reader, and make adaptations to allow a clearer link to the standard two-sided classical sequent calculus.

The basic idea is very simple. A formula is represented as a two-dimensional 'matrix' of its constituent literals, the structure of the matrix being determined by the occurrences of the connectives in the formula. The matrix itself is built hierarchically, using at each stage either a horizontal or vertical vector of other matrices. The idea is illustrated by an example taken from Wallen [21].

Consider the formula

$$
\phi=((\mathrm{p} \supset \mathrm{q}) \wedge(\mathrm{q} \supset \mathrm{r})) \supset(\mathrm{p} \supset \mathrm{r}),
$$

This is equivalent to $(\mathrm{p} \wedge \neg \mathrm{q}) \vee(\mathrm{q} \wedge \neg \mathrm{r}) \vee(\neg \mathrm{p} \vee \mathrm{r})$.

The matrix representation of this is in turn

$$
\left(\begin{array}{c}
\mathrm{p} \\
\neg \mathrm{q}
\end{array}\right) \quad\left(\begin{array}{c}
\mathrm{q} \\
\neg \mathrm{r}
\end{array}\right) \quad(\neg \mathrm{p} \quad \mathrm{r})
$$

It will be observed that this is a horizontal row matrix, the horizontality corresponding to disjunction. The first two components of this are vertical vectors (conjunction) with the third horizontal (disjunction again).

Given a matrix representation of a formula, we determine whether the formula is provable by considering paths through the matrix, and the span of the set of connections in the matrix: 
- A path through the matrix is a sequence of literals that, read from left to right, contains a element from each column of the matrix. In our example, the paths are

$$
\begin{array}{ll}
\{\mathrm{p}, \mathrm{q}, \neg \mathrm{p}, \mathrm{r}\} & \{\neg \mathrm{q}, \mathrm{q}, \neg \mathrm{p}, \mathrm{r}\} \\
\{\mathrm{p}, \neg \mathrm{r}, \neg \mathrm{p}, \mathrm{r}\} & \{\neg \mathrm{q}, \neg \mathrm{r}, \neg \mathrm{p}, \mathrm{r}\}
\end{array}
$$

- The connections in the matrix are the pairs of complementary literals that occur in the matrix. In our example, the pairs are

$$
\{\mathrm{p}, \neg \mathrm{p}\} \quad\{\neg \mathrm{q}, \mathrm{q}\} \quad\{\neg \mathrm{r}, \mathrm{r}\}
$$

- A set of connections spans the formula if every path contains a connection from the set. In our example, the connections do indeed span the formula.

If the set of connections spans the formula, then the formula is provable. Conversely, if we can find a path that contains no connection (no complementary pair of literals), then the formula is unprovable. Thus provability is characterized by the existence of spanning sets of connections.

The importance of the matrix method in automated theorem proving derives from its efficiency, both as practical basis for theorem provers, and as an elegant representation of proofs in which redundancy in the search space is, essentially, eliminated [21].

We now give a more formal account of the method. As indicated in Bibel [4], we give two translations for each formula, a positive and a negative (essentially the translation of the negation).

\begin{tabular}{|l|c|c|c|}
\hline & $\varphi$ & $\llbracket \varphi \rrbracket^{+}$ & $\llbracket \varphi \rrbracket^{-}$ \\
\hline literal & $P$ & $P$ & $\neg P$ \\
\hline negated literal & $\neg P$ & $\neg P$ & $P$ \\
\hline conjunction & $\varphi \wedge \psi$ & $\begin{array}{c}\llbracket \varphi \rrbracket^{+} \\
\llbracket \psi \rrbracket^{+}\end{array}$ & $\llbracket \varphi \rrbracket^{-} \llbracket \psi \rrbracket^{-}$ \\
\hline disjunction & $\varphi \vee \psi$ & $\llbracket \varphi \rrbracket^{+} \llbracket \psi \rrbracket^{+}$ & $\begin{array}{c}\llbracket \varphi \rrbracket^{-} \\
\llbracket \psi \rrbracket^{-}\end{array}$ \\
\hline implication & $\varphi \supset \psi$ & $\llbracket \varphi \rrbracket^{-} \llbracket \psi \rrbracket^{+}$ & $\begin{array}{c}\llbracket \varphi \rrbracket^{+} \\
\llbracket \psi \rrbracket^{-}\end{array}$ \\
\hline negation & $\neg \varphi$ & $\llbracket \varphi \rrbracket^{-}$ & $\llbracket \varphi \rrbracket^{+}$ \\
\hline true & $\top$ & $\top$ & $($ blank) \\
\hline false & $\perp$ & (blank) & $\top$ \\
\hline
\end{tabular}

The fact that no brackets are used around the vectors is intended to indicate that successive rows or columns are merged, so that $\llbracket(A \vee B) \vee C \rrbracket^{+}$is the flat row matrix with three elements: $(A B C)$, not a nested structure: $((A B) C)$. 
Sequents can be translated by equating $\Gamma_{1} \ldots \Gamma_{m} \vdash \Delta_{1} \ldots \Delta_{n}$ with the formula $\left(\Gamma_{1} \wedge \ldots \wedge \Gamma_{m}\right) \supset\left(\Delta_{1} \vee \ldots \vee \Delta_{n}\right)$, so that $\llbracket \Gamma_{1} \ldots \Gamma_{m} \vdash \Delta_{1} \ldots \Delta_{n} \rrbracket^{+}$is

$$
\llbracket \Gamma_{1} \rrbracket^{-} \ldots \llbracket \Gamma_{m} \rrbracket^{-} \llbracket \Delta_{1} \rrbracket^{+} \ldots \llbracket \Delta_{n} \rrbracket^{+}
$$

and $\llbracket \Gamma_{1} \ldots \Gamma_{m} \vdash \Delta_{1} \ldots \Delta_{n} \rrbracket^{-}$is a similar column.

Paths through these matrices are defined inductively on the structure of the matrix. We write $\{M \mid\}$ for the set of paths through a matrix $M$.

If $M$ is atomic, (in the case of the matrix arising from a formula, this is when the formula is a literal or negated literal), then $\{|M|\}=\{[A]\}$, where $A$ is the atomic element of $M$.

If $M$ is a horizontal vector: $M=M_{1} \ldots M_{n}$, then

$$
\{|M|\}=\left\{P_{1} @ \ldots @ P_{n} \mid P_{1} \in\left\{M_{1} \mid\right\}, \ldots, P_{n} \in\left\{\left|M_{n}\right|\right\}\right\},
$$

the result of all possible concatenations of paths through each component.

$$
\begin{aligned}
& M_{1} \\
& \text { If } M \text { is a vertical vector: } M=\vdots \text {, then } \\
& M_{n} \\
& \{M \mid\}=\left\{\left|M_{1}\right|\right\} \cup \ldots \cup\left\{\left|M_{n}\right|\right\},
\end{aligned}
$$

the result of choosing a path through a component.

In the case that $\varphi$ is a formula, or sequent, we write $\{|\varphi|\}^{+}$for the result of taking paths through $\llbracket \varphi \rrbracket^{+}$, and similarly $\{|\varphi|\}^{-}$for the result of taking paths through $\llbracket \varphi \rrbracket^{-}$.

Note that while a matrix is essentially tree-structured, the set of paths is always a set of lists of atomic entries in the matrix (leaves of the tree). In essence, taking the set of paths of the matrix associated with a formula equates to putting the formula in conjunctive normal form. The formula is therefore valid if and only if each path is, and hence we have:

Theorem 4. $\varphi$ is valid if and only if each path in $\{|\varphi|\}^{+}$contains $T$ or at least one pair of complementary literals, $A$ and $\neg A$.

In theorem-proving applications, it is reasonable to stop at this point. But we will make a finer analysis based on proof theory.

For all rules of LK except for cut and weakening, any occurrence of a literal as part of a formula in the consequence of the rule can be traced back to one or more occurrences in the premisses. It follows that if we have a cut-free proof of a sequent, then certain complementary pairs of literals in that sequent trace back to the complementary pair arising from one of the axioms in the proof. In fact:

Theorem 5. Suppose $P$ is a cut-free proof in the propositional fragment of $L K$ of the sequent $\Gamma \vdash \Delta$, then each path in $\{\mid \Gamma \vdash \Delta\}\}^{+}$contains $T$ or contains a complementary pair of literals arising from an axiom $A \vdash A$ in the proof. 
The proof is a straightforward induction on the structure of $P$.

What this theorem tells us is that the axioms used in a proof give us a token in the form of a set of pairs of leaves of the matrix that suffices to establish validity of the sequent, and what the matrix condition tells us is that we have an abstract condition on a set of pairs of leaves that tells us that the set is sufficient to justify validity of the formula. We can think of such a set as the ghost of a proof. We take this thought further and express it in a more combinatorial fashion in the next section where we show abstractly that these ghosts can be combined using the same rules as conventional proofs, and hence furnish a model of proof theory and not just validity.

\section{A Combinatorial Model}

In this section, we set up a purely combinatorial framework corresponding to the matrix technology discussed above. We start with a set of atoms, $\mathcal{A}$, corresponding to the atoms (positive literals) in the matrix arising from a formula. Our first two definitions merely bring home the point that the matrices and paths used in the matrix method can be defined in a purely combinatorial setting.

Definition 4. The set of matrices over $\mathcal{A}$ is obtained inductively, with each matrix derived as one of

1. an atomic matrix $A$ corresponding to an element of $\mathcal{A}$,

2. an atomic matrix $\neg A$ corresponding to the formal negation of an element of $\mathcal{A}$,

3. the horizontal composite of a finite (possibly empty) sequence of matrices $M_{1} \oplus \ldots \oplus M_{n}$, and

4. the vertical composite of a finite (possibly empty) sequence of matrices $M_{1} \otimes$ $\ldots \otimes M_{n}$.

There are a number of design decisions here. Our matrices are either atomic, or a horizontal composite (corresponding to a disjunction) or a vertical composite (corresponding to a conjunction). We have not made the assumption that a horizontal composite only contains vertical composites and atomic matrices. This is a difference with the presentation of Bibel's method, where we were careful to flatten consecutive sums and (or respectively products) into a single multiple sum (or product), so that we always had $\left(M_{1} \oplus M_{2} \oplus M_{3}\right)$ and not $\left(M_{1} \oplus\left(M_{2} \oplus M_{3}\right)\right.$ ). Putting this requirement in would be an option, but slightly complicate the inductive definition. The resulting structures would be equivalent, and have the same sets of paths.

A further choice is that, motivated by indexed type theory, we treat $T$ as the empty conjunction, and $\perp$ as the empty disjunction. There is a difference here with the standard presentation of the matrix method. This would have $T$ and $\perp$ as constant atomic matrices. There is a difference here that shows up in the paths through the matrices (taking $T$ as the empty sum means we have fewer paths). Our validity condition would then change from 'every path contains a 
pair of complementary literals' to 'every path contains a pair of complementary literals or T'. This choice is less uniform, but it means that we are not throwing information away if we take a disjunction with $T$.

We view these matrices as trees, and will be interested in relations on the leaves. The leaves are either atomic matrices or empty products or sums.

We now formally define the notion of paths through a matrix.

Definition 5. The set of paths through a matrix $M,\{|M|\}$, is defined through induction on the substructures $M^{\prime}$ of $M$ as follows:

1. if $M^{\prime}$ is a leaf consisting of the atomic matrix corresponding to an element $A$ of $\mathcal{A}$, then $\{|M|\}=\left\{\left[A_{p}\right]\right\}$, where the annotation $p$ gives the position of the leaf in $M$;

2. if $M^{\prime}$ is the empty horizontal composite, then $\left\{\mid M^{\prime}\right\}=\{[]\}$; that is, the only path through $M^{\prime}$ is the empty sequence;

3. if $M^{\prime}$ is a non-empty horizontal composite, $M^{\prime}=M_{1} \oplus \ldots \oplus M_{n}$, then $\left\{\left|M^{\prime}\right|\right\}=\left\{P_{1} @ \ldots @ P_{n} \mid P_{1} \in\left\{\left|M_{1}\right|\right\}, \ldots, P_{n} \in\left\{\left|M_{n}\right|\right\}\right\}$, the result of all possible concatenations of paths through each component;

4. if $M^{\prime}$ is the empty vertical composite, then $\left\{M^{\prime}\right\}=\emptyset$; that is, there are not paths through the empty vertical composite;

5. if $M^{\prime}$ is a non-empty vertical composite, $M^{\prime}=M_{1} \otimes \ldots \otimes M_{n}$, then $\left\{\mid M^{\prime}\right\}=$ $\left\{\left|M_{1}\right|\right\} \cup \ldots \cup\left\{M_{n} \mid\right\}$.

The set of paths is once again quite a flat structure; it is a set of sequences, each of which is a sequence containing elements of $\mathcal{A}$.

It is worth spending a little more time on our treatment of empty composites. Our validity condition requires that all paths contain elements with particular properties. There are no paths through an empty vertical composite, and hence this condition is vacuously satisfied. This is what you would expect from the representation of $T$. Similarly, the empty horizontal composite $(\perp)$ has one path through it, and this can never satisfy our condition. Hence $\perp$ is impossible to prove.

The next definition translates the terminology of Definition 2.1 from Bibel [4] into our slightly more general setting.

Definition 6. A set of connections or links for a matrix $M$ over $\mathcal{A}$ is a set of unordered pairs of leaves of $M$, each of which relates two complementary atomic matrices. A set $S$ of connections is said to span $M$ if every path in $M$ contains a pair of leaves from $S$. A set of connections, $S$, is said to be a relator for $M$ if it spans $M$.

Examples:

(i) Consider the formula

$$
\phi=\neg \mathrm{p} \vee \neg \mathrm{q} \vee(\mathrm{p} \wedge \mathrm{q}),
$$

with associated matrix $M=\neg \mathrm{p} \oplus \neg \mathrm{q} \oplus(\mathrm{p} \otimes \mathrm{q})$, and paths

$$
\{|M|\}=\{\{\neg \mathrm{p}, \neg \mathrm{q}, \mathrm{p}\},\{\neg \mathrm{p}, \neg \mathrm{q}, \mathrm{q}\}\} .
$$


The relation that links $\neg \mathrm{p}$ with $\mathrm{p}$ and $\neg \mathrm{q}$ and $\mathrm{q}$ is a relator for $M$ (or by abuse of language, $\phi)$.

(ii) Take $\psi=(\neg \mathrm{p} \wedge \neg \mathrm{q}) \vee(\mathrm{p} \wedge \mathrm{q})$, which has associated matrix $N=(\neg \mathrm{p} \oplus \neg \mathrm{q}) \oplus$ $(\mathrm{p} \otimes \mathrm{q})$. Then

$$
\{N \mid\}=\{\{\neg \mathrm{p}, \mathrm{p}\},\{\neg \mathrm{p}, \mathrm{q}\},\{\neg \mathrm{q}, \mathrm{p}\},\{\neg \mathrm{q}, \mathrm{q}\}\} .
$$

We see that there is no relator for $N(\psi)$ because there is no possible relation between literals for either of the sets $\{\neg \mathrm{p}, \mathrm{q}\}$ and $\{\neg \mathrm{q}, \mathrm{p}\}$.

(iii) On the other hand, if $\chi=\neg \mathrm{p}^{1} \vee \neg \mathrm{p}^{2} \vee\left(\mathrm{p}^{1} \wedge \mathrm{p}^{2}\right)$, with associated matrix $P=$ $\neg \mathrm{p}^{1} \oplus \neg \mathrm{p}^{2} \oplus\left(\mathrm{p}^{3} \oplus \mathrm{p}^{4}\right)$, we have $\{|P|\}=\left\{\left\{\neg \mathrm{p}^{1}, \neg \mathrm{p}^{2}, \mathrm{p}^{3}\right\},\left\{\neg \mathrm{p}^{1}, \neg \mathrm{p}^{2}, \mathrm{p}^{4}\right\}\right\}$. There are several relators for $P(\chi)$. One links $\mathrm{p}^{3}$ and $\neg \mathrm{p}^{1}$ and $\mathrm{p}^{4}$ and $\neg \mathrm{p}^{2}$. Another one links $\mathrm{p}^{3}$ and $\neg \mathrm{p}^{2}$ and $\mathrm{p}^{4}$ and $\neg \mathrm{p}^{2}$.

(iv) Lastly, consider Peirce's law, $\pi=((\mathrm{p} \supset \mathrm{q}) \supset \mathrm{p}) \supset \mathrm{p}$, which translates into our setting as $Q=\left(\left(\neg \mathrm{p}_{1} \oplus \mathrm{q}_{2}\right) \otimes \neg \mathrm{p}_{3}\right) \oplus \mathrm{p}_{4}$. The associated set of paths $\{|Q|\}$ is

$$
\left\{\left\{\neg \mathrm{p}_{1}, \mathrm{q}_{2}, \mathrm{p}_{4}\right\},\left\{\neg \mathrm{p}_{3}, \mathrm{p}_{4}\right\}\right\},
$$

which has a relator which links both $\neg \mathrm{p}_{1}$ and $\neg \mathrm{p}_{3}$ with $\mathrm{p}_{4}$.

If we view matrices as our semantics of propositions, then we also view relators as our semantics of proofs. It is immediate from the definition of relator that each relator gives a certificate that the matrix method will succeed in showing the corresponding proposition's provability. And we have noted earlier that cut-free proofs generate relators. We will now go further and show that relators function well as an abstract interpretation of proofs. This is demonstrated through a number of technical properties. First the sequent proof rules can be interpreted in matrices and relators. Second, any relator contains, in a certain sense, a proof. Finally, we shall see that matrices and relators give rise to a classical category as in $\S 2$.

The following lemma makes a number of obvious identifications for the sake of clarity of exposition.

Lemma 1. (i) The matrices $M \oplus(N \oplus P)$ and $(M \oplus N) \oplus P$ have the same set of leaves and the same set of paths, and hence any relator for one is a relator for the other (and similarly for $M \otimes(N \otimes P)$ ).

(ii) The matrices $M \oplus N$ and $N \oplus M$ have paths which are simply re-orderings of each other, and hence any relator for one is a relator for the other.

(iii) The paths for $M \oplus(N \otimes P)$ are the disjoint union of the paths for $M \oplus N$ and $M \oplus P$.

(iv) If $S$ is a relator for $M \otimes(N \otimes P)$, then its restriction to $M \otimes N$ is a relator for $M \otimes N$, and similarly for $M \otimes P$.

(v) Conversely, if $S_{1}$ is a relator for $M \otimes N$ and $S_{2}$ is a relator for $M \otimes P$ then $S_{1} \cup S_{2}$ is a relator for $M \otimes(N \otimes P)$.

(vi) If $S$ is a relator for $M$, then $S$ is also a relator for $M \oplus N$.

(vii) If $S$ is a relator for $M \oplus N \oplus N$, then the relator $\bar{S}$ obtained by identifying corresponding literals in the two occurrences of $N$ is a relator for $M \oplus N$. 
Proof. Direct consequences of the definition of relators.

In $\S 3$ above we discussed the translation of sequents into formulae, and then the interpretation of formulae as matrices. Under this interpretation, the twosided sequent rules collapse as follows. Axiom and cut translate to single-sided versions. Each of the left-handed structural rules becomes the corresponding right-handed rule. The negation rules (left and right) become vacuous since their conclusion is identical to their hypothesis. $(\perp L)$ becomes $(\top R) .(\wedge L)$ and $(\vee L)$ become, respectively, $(\vee R)$ and $(\wedge R)$. $(\supset L)$ becomes $(\wedge R)$ and $(\supset R)$ trivializes in the same way as the negation rules. After all this, the rules are as follows.

The axiom and cut rules are, respectively,

$$
\overline{\vdash \mathrm{p}, \neg \mathrm{p}} \text { Axiom and } \frac{\vdash \Gamma, \phi \quad \vdash \neg \phi, \Delta}{\vdash \Gamma, \Delta} \mathrm{Cut},
$$

the operational rules are

$$
\frac{\vdash \phi, \psi, \Gamma}{\vdash \phi \vee \psi, \Gamma} \vee \quad \text { and } \quad \frac{\vdash \phi, \Gamma \vdash \psi, \Delta}{\vdash \phi \wedge \psi, \Gamma, \Delta} \wedge, \quad \text { with unit } \frac{\vdash \top}{\vdash \top}^{\top},
$$

and the structural rules of exchange, contraction and weakening are, respectively

$$
\frac{\vdash \phi, \psi, \Gamma}{\vdash \psi, \phi, \Gamma} E, \quad \frac{\vdash \phi, \phi, \Gamma}{\vdash \phi, \Gamma} C, \text { and } \frac{\vdash \Gamma}{\vdash \phi, \Gamma} W .
$$

These can be interpreted in matrices and relators. Conjunction is interpreted as $\otimes$, and disjunction as $\oplus$, with $T$ as the empty product. Negation is interpreted in the obvious way as the operation that keeps the basic tree structure, interchanging $\oplus$ and $\otimes$ and negating all of the atoms. To make this clear we write $S \vdash M$ for " $S$ is a relator for $M$ ". We will also abuse notation by writing elements or relators as ordered, instead of unordered, pairs.

The cut-free calculus can be interpreted in matrices and relators.

Lemma 2. (i) (Axiom) If $A$ is an atom, then $\{(A, \neg A)\} \vdash A \oplus \neg A$.

(ii) ( $V$ ) If $S \vdash M \oplus N \oplus P$, then $S^{*} \vdash(M \oplus N) \oplus P$ (and conversely), where $S^{*}$ is the translation of $S$ through the canonical isomorphism between $M \oplus N \oplus P$ and $(M \oplus N) \oplus P$.

(iii) ( $\wedge$ If $R \vdash M \oplus P$ and $S \vdash N \oplus Q$ then $R \cup S \vdash(M \otimes N) \oplus P \oplus Q$

(iv) (Unit) The empty set of pairs is a relator for the empty product of matrices: $\emptyset \vdash \top$.

(v) (Exchange) If $S \vdash M \oplus N \oplus P$, then also $S^{\prime} \vdash N \oplus M \oplus P$, where $S^{\prime}$ is the translation of $S$ through the canonical isomorphism.

(vi) (Contraction) If $S \vdash M \oplus N \oplus N$, then the relator $\bar{S}$ obtained by identifying corresponding literals in the two occurrences of $N$ is a relator for $M \oplus N$, so $\bar{S} \vdash M \oplus N$.

(vii) (Weakening) If $S \vdash M$, then also $S \vdash M \oplus N$.

Proof. All follow easily from basic properties of relators and the corresponding statements in Lemma 1. 
The interpretation of the Cut rule is more problematic. While it was easy to give direct constructions for the relators in the other rules, Cut requires a more complex construction. We give first an inductive definition that will justify the admissibility of Cut.

Definition 7. If $R$ is a relator for $N \oplus M$ and $S$ one for $\neg M \oplus P$, then we construct a set of pairs of complementary literals, cut $(M ; R, S)$, for $N \oplus P$ as follows:

(i) if $M$ is the atomic matrix corresponding to the literal $A$ then take cut $(M ; R, S)$ to be the set of unordered pairs:

$$
\begin{gathered}
\left\{\left(n, n^{\prime}\right) \in R \mid n, n^{\prime} \in N\right\} \cup\left\{\left(p, p^{\prime}\right) \in R \mid p, p^{\prime} \in P\right\} \\
\cup\{(n, p) \mid n \in N \wedge p \in P \wedge(n, A) \in R \wedge(\neg A, p) \in S\}
\end{gathered}
$$

In other words, in addition to those pairs from $N$ that are linked by $R$ and those from $P$ that are linked by $S$, which we inherit from the constituent relators, we also include pairs of elements from the composite $R I_{A} S$, where $I d_{A}$ is the relation linking $A$ to $\neg A$.

(ii) if $M$ is the empty sum of matrices, then we take $\operatorname{cut}(M ; R, S)=R$

(iii) if $M$ is a non-empty sum $M=M_{1} \oplus \ldots \oplus M_{n}$, then by Lemma 1 we can restrict $S$ to relators $S_{i} \vdash \neg M_{i} \oplus Q$, and consider $\operatorname{cut}\left(M_{n} ; \ldots \operatorname{cut}\left(M_{2} ; \operatorname{cut}\left(M_{1} ; R, S_{1}\right), S_{2}\right) \ldots, S_{n}\right)$, which is a set of complementary literals of $N \oplus P \oplus \ldots \oplus P$. We define $\operatorname{cut}(M ; R, S)$ to be the result of quotienting this down to $N \oplus P$

(iv) if $M$ is a negated literal, or product of matrices, then $\neg M$ is an unnegated literal or sum of matrices and we define $\operatorname{cut}(M ; R, S)=\operatorname{cut}(\neg M ; S, R)$.

Lemma 3. If $R$ is a relator for $N \oplus M$ and $S$ one for $\neg M \oplus P$, then $\operatorname{cut}(M ; R, S)$ is a relator for $N \oplus P$.

Proof. We prove this by induction on the structure of $M$.

(i) Suppose $M$ is an atomic matrix $A, R$ is a relator for $N \oplus A$ and $S$ a relator for $\neg A \oplus P$, we show that $\operatorname{cut}(A ; R, S)$ is a relator for $N \oplus P$. Suppose we are given a path $\nu$ in $N$, and a path $\pi$ in $\mathrm{P}$. We show that the concatenation of the two always contains a pair from $\operatorname{cut}(A ; R, S)$. If $\nu$ contains a pair from $R$, or $\pi$ contains a pair from $S$, then this is trivially the case. Suppose now that neither $\nu$ or $\pi$ contains such a pair. Consider the path $\nu @[A]$ in $N \oplus A$. Since $R$ is a relator, it must contain a pair on this path, which now must be $(n, A)$ for some $n$ on $\nu$. Similarly $S$ must contain a pair $(\neg A, p)$ for some $p$ on $\pi$. Therefore $\operatorname{cut}(A ; R, S)$ contains the pair $(n, p)$ on $\nu @ \pi$ as required.

(ii) Suppose $M=\perp$ is the empty sum. If $R$ is a relator for $N \oplus \perp$, then $\operatorname{cut}(\perp ; R, S)=R$ is also a relator for $N$, and hence for $N \oplus P$.

(iii) We give detail for the binary case. The general case follows by induction. Suppose $M=M_{1} \oplus M_{2}$, then by induction, using the same notation as the definition, $\operatorname{cut}\left(M_{1} ; R, S_{1}\right)$ is a relator for $N \oplus M_{2} \oplus P$, and $\operatorname{cut}\left(M_{2} ; \operatorname{cut}\left(M_{1} ; R, S_{1}\right), S_{2}\right)$ is a relator for $N \oplus P \oplus P$. Hence, by lemma $1, \operatorname{cut}(M ; R, S)$ is a relator for $N \oplus P$. 
(iv) Suppose $M$ is a negated literal, or product of matrices, then $\neg M$ is a positive literal or sum of matrices, and hence fits the conditions of the previous cases. The result now follows because of the commutavity of $\oplus$ for relators as in Lemma 1.

We now have interpretations of all the rules of the sequent calculus. From this we get soundness for provability.

Proposition 1 (soundness for provability). Let $\mathcal{A}$ be a set of atoms, and let $\phi$ be any propositional formula with atoms in $\mathcal{A}$. Let $M$ be the matrix over atoms $\mathcal{A}$ associated with $\phi$. If $\Phi$ is a proof in the sequent calculus of $\phi$, then there is a set of complementary literals $R^{\Phi}$ for $M$, functional in both $R$ and $\Phi$ that is a relator for $\phi$.

Lemma 3 implicitly gives an algorithm for the cut operation recursive on the structure of the matrix being cut. Before going further we sketch out what this means for a couple of small examples. We use an obvious compositional notation. Example 1: $M=A \oplus B$, where $A$ and $B$ are atomic. In this case, $\operatorname{cut}(M ; R, S)$ resolves to the union of:

$$
\begin{gathered}
\left\{\left(n, n^{\prime}\right) \in R\right\} \\
\left\{\left(p, p^{\prime}\right) \in S\right\} \\
\{(n, p) \mid n R A(\neg A) S p \vee n R B(\neg B) S p\} \\
\left\{\left(p, p^{\prime}\right) \mid p S(\neg B) B R A(\neg A) S p^{\prime}\right\}
\end{gathered}
$$

Since these pairs are treated as unordered, this is symmetric in $A$ and $B$ despite our choice of cutting $A$ first. The first two components are simply pairs inherited from $R$ and $S$. The third is the obvious composite through the atomic elements of the cut term. In the final entry, however, we get extra pairs in $P$ obtained by passing from $P$ through $S$ to $B$, then through $R$ to $A$, if this is possible, and finally back through $S$ to $P$ again. We do not get the analogous pairs in $N$.

Example 2: $M=\left(A_{1} \otimes A_{2}\right) \oplus B$, where all the elements are atomic. We first compute $\operatorname{cut}\left(\left(A_{1} \otimes A_{2}\right) ; R, S\right)$. This is a relator on $N \oplus B \oplus P$ linking the outer elements of composites of the form: $(n / B) R n^{\prime}, p S p^{\prime},(n / B) R A_{i}\left(\neg A_{i}\right) S p$, and $(n / B) R A_{i}\left(\neg A_{i}\right) S\left(\neg A_{j}\right) A_{j} R n^{\prime}$. We then eliminate the cut on $B$ to get a relator linking the outer elements of the composites:

$$
\begin{gathered}
n R n^{\prime}, p S p^{\prime}, n R A_{i}\left(\neg A_{i}\right) S p, n R B(\neg B) S p, \\
n R A_{i}\left(\neg A_{i}\right) S\left(\neg A_{j}\right) A_{j} R n^{\prime}, p S\left(\neg A_{i}\right) A_{i} R B(\neg B) S p, \\
\quad \text { and } n R A_{i}\left(\neg A_{i}\right) S\left(\neg A_{j}\right) A_{j} R B(\neg B) S p .
\end{gathered}
$$

The last of these shows a significant path inside the term being cut.

We now turn our attention to the completeness of the structure. We will show that any relator contains one derived from a cut-free proof.

Proposition 2 (completeness). If $\mathcal{A}$ is a set of atoms, $M$ is a matrix over $\mathcal{A}$ and $R$ is a relator for the matrix $M, R \vdash M$, then there is a cut-free sequent derivation $\Phi$ of $M$ such that $R^{\Phi} \subseteq R$. 
Proof. The proof uses the structure of $M$. We use the following versions of the sequent rules to work backwards from $M$ to construct a derivation:

$$
\begin{array}{cc}
\frac{S \vdash M \oplus N \oplus P}{S \vdash(M \oplus N) \oplus P} \vee & \frac{S_{M} \vdash M \oplus P \quad S_{N} \vdash N \oplus P}{S \vdash(M \otimes N) \oplus P} \wedge \\
\frac{S \vdash M}{S \vdash \perp \oplus M} \perp & \overline{S \vdash \top \oplus M}^{\top}
\end{array}
$$

These rules are all admissible. We continue until either we reach a term of the form $\top \oplus M$ or one of the form a sum of literals. At each stage we have a valid relator for the remaining term, and at each stage the relator is a subset of the original relator $R$ (for this reason we cannot end up with a proof of $\perp$ or a relator for an empty term). In the case that we have a sum of literals, then at least one pair of literals, $A$ and $\neg A$ must be contained in the relator at that point. We therefore have $S \vdash A \oplus \neg A \oplus N$, where $(A, \neg A) \in S$ and $S \subseteq R$. We now replace this with the proof fragment

$$
\frac{\overline{\{(A, \neg A)\} \vdash A \oplus \neg A} \text { Axiom }}{\{(A, \neg A)\} \vdash A \oplus \neg A \oplus N} W
$$

We continue reversing our deconstruction of $M$ to build a derivation along with its associated relator. There is no difficulty with sums, but products require contraction:

$$
\frac{\frac{S \vdash N \oplus Q \quad S^{\prime} \vdash P \oplus Q}{S \cup S^{\prime} \vdash(N \otimes P) \oplus Q \oplus Q}}{S \cup S^{\prime} \vdash(N \otimes P) \oplus Q} \wedge
$$

At each point in this construction, we have a relator which is contained in our original relator $R$, and hence the result follows.

Relators thus contain the ghost of (at least one) cut-free derivation of the matrix, and can thus be thought of as a proof plus junk. But some possible terms are more junk than others. The relators derived from cut-free proofs contain only pairs that live on the same path. At first sight it looks as if the cut operation we gave above can break this property so that relators derived from proofs containing cut can contain pairs not on the same path. However, this is not the case:

Lemma 4. Suppose $\Phi$ is a derivation of the matrix $M$, possibly containing cut, then any pair $(p, \neg p)$ of complementary literals in $R^{\Phi}$ can be found in some path through $M$.

Proof. We need only check the cut rule. This follows by induction on the structure of the matrix being cut. It is easily checked when the matrix is atomic. But when it is not, the cut is obtained as the cut of two smaller matrices, and hence the result follows by induction. 


\section{A Second Interpretation of Cut}

Semantically there is more than one interpretation of Cut. This is not a surprise from a logical perspective, logicians are accustomed to the fact that there is more than one process for eliminating cut from proofs. However, not all semantic interpretations correspond to logical processes of cut elimination. Indeed the interpretation given above when applied to the Lafont example results in the union of the two relators, and hence to a relator that does not correspond to a single cut-free proof.

In this section we give a second interpretation of Cut. The original inductive interpretation is useful for establishing validity, but it is difficult to visualize and reason with. The second interpretation given in this section is more geometric and combinatorial.

We motivate this new definition through a further analysis of the inductive definition given above.

Any relator is a set of unordered pairs of literals in the matrix, and as such can be viewed as an undirected graph on the set of literals. Since relators link literals with their negations, this is, in fact, a bipartite graph.

When we have $R \vdash N \oplus M$ and $S \vdash \neg M \oplus P$, we construct $\operatorname{cut}(M ; R, S)$ by considering paths through a graph on $N \oplus M \oplus \neg M \oplus P$, specifically the graph $R \cup 1_{M} \cup S$. Here $1_{M}$ is the graph linking corresponding pairs of literals in $M$ and $\neg M$ (and which will be the identity on $M$ in the eventual categorical model). $\operatorname{cut}(M ; R, S)$ is then the relator linking the endpoints of a certain set of paths in this graph.

Lemma 5. Any element of $\operatorname{cut}(M ; R, S)$ is a pair of literals linked by a path with the following properties:

(i) The path begins (and ends) with a link from $R \cup S$;

(ii) Links from $R \cup S$ alternate with links from $1_{M}$;

(iii) The path stays in $M \oplus \neg M$ except for the first and last links;

(iv) The path satisfies the regular expression

$$
R\left(1_{M} S 1_{M} R\right)^{*}+R\left(1_{M} S 1_{M} R\right)^{*} 1_{M} S+S\left(1_{M} R 1_{M} S\right)^{*}
$$

Proof. In general, the proof is by induction on the structure of $M$. However, property (i) follows simply from the pair of literals being linked by a path, property (iii) follows from property (ii), and property (iv) from (i)-(iii).

This lemma motivates our revised interpretation of cut.

Definition 8. If $R \vdash N \oplus M$ and $S \vdash \neg M \oplus P$, then $\operatorname{Cut}(M ; R, S)$ (note the capitalization) is the restriction to $N \oplus P$ of

$$
R\left(1_{M} S 1_{M} R\right)^{*}+R\left(1_{M} S 1_{M} R\right)^{*} 1_{M} S+S\left(1_{M} R 1_{M} S\right)^{*}
$$

Equivalently, $\operatorname{Cut}(M ; R, S)$ is the set of endpoints of paths from $N$ to $N$ or $P$, or from $P$ to itself, which have odd length, start with a link from $R$ or $S$, and in which links from $R$ and $S$ alternate with links from $1_{M}$. 
Lemma 6. If $R \vdash N \oplus M$ and $S \vdash \neg M \oplus P$, then $\operatorname{Cut}(M ; R, S) \vdash N \oplus P$.

Proof. This follows immediately from Lemma 3 , since $\operatorname{Cut}(M ; R, S)$ is a superset of $\operatorname{cut}(M ; R, S)$.

Hence $\operatorname{Cut}(;,$,$) is also a valid interpretation of the Cut rule. Note in par-$ ticular that if $R \vdash N \oplus \perp$ and $S \vdash \top \oplus P$, then $\operatorname{Cut}(M ; R, S)=R \cup S$.

\section{The Classical Category}

In this section, we show that matrices and relators form a classical category, $\mathcal{C P}$, as in $\S 2$.

The objects of $\mathcal{C P}$ are matrices, and the morphisms from a matrix $M$ to a matrix $N$ are relators $R \vdash \neg M \oplus N$. The ordering on morphisms is reverse inclusion of relations, so that $R \leq S$ iff $S \subseteq R$. As expected, composition is given by the second interpretation of Cut, as in $\S 5$. The identity on a matrix $M$ is the relator $1_{M} \vdash \neg M \oplus M$ as in $\S 5$.

We will make substantial use of a technical lemma.

Lemma 7. Suppose $R \vdash \neg N \oplus M$ and $S \vdash \neg M \oplus P$, and that $R$ is a bijection between the literals of $\neg N$ and the literals of $M$, then $\operatorname{Cut}(M ; R, S)$ is the relator obtained from $S$ by substituting literals of $\neg N$ for literals of $\neg M$ along the bijection obtained from $R$.

Proof. Consider the paths through $M \oplus \neg M$ in which $1_{M}$ alternates with $R \cup S$. There can be no links from $R$, since all such go to $\neg N$, and hence any such path must be contained in a string from $1_{M} S 1_{M}$. It follows that the elements of $\operatorname{Cut}(M ; R, S)$ are the elements of $S$ linking literals in $P$, and the result of substituting the appropriate elements of $N$ in elements of $S$ which link $\neg M$ to $P$, or $\neg M$ to itself, as required.

There is an obvious analogue of this result for composition on the right, and also an obvious generalization for relators $R$ that contain no links from $M$ to itself.

Lemma 8. $\mathcal{C P}$ is an order-enriched category.

Proof. Suppose $R, S$ and $T$ are relators that can be composed in $\mathcal{C P}$ :

$$
R \vdash \neg N \oplus M \quad S \vdash \neg M \oplus P \quad T \vdash \neg P \oplus Q
$$

We view the composite as given by the endpoints of a set of paths in an undirected graph on (the literals of) $\neg N \oplus M \oplus \neg M \oplus P \oplus \neg P \oplus Q$. Then $\operatorname{Cut}(M ; R, S)$ is the set of paths from $R \cup S \cup 1_{M}$, of odd length, starting with a link from $R \cup S$, and in which links from $R \cup S$ alternate with links from $1_{M}$. We claim that $\operatorname{Cut}(P ; \operatorname{Cut}(M ; R, S), T)$ is the set of paths of odd length, starting with a link from $R \cup S \cup T$ and in which links from $R \cup S \cup T$ alternate with links 
from $1_{M} \cup 1_{P}$. The symmetry of this characterization implies immediately that composition is associative.

Proof of claim: $\operatorname{Cut}(P ; \operatorname{Cut}(M ; R, S), T)$ can be characterized as the result of taking end-points of paths of odd length, each component of which is an element of $\operatorname{Cut}(M ; R, S) \cup T \cup 1_{P}$, with the elements of $\operatorname{Cut}(M ; R, S)$ considered as a single step. If we now expand these elements to be subpaths, we are replacing a single step by an odd number, and hence increasing the total length of the path by an even number of steps. The total length therefore remains odd. Moreover the alternation condition remains valid. Conversely, if we have a path in which links from $R \cup S \cup T$ alternate with links from $1_{M} \cup 1_{P}$, then we can consider the subpaths between consecutive links from $1_{P}$. If the subpath contains any link from $T$, then, since $T \vdash \neg P \oplus Q$, the link either goes to $Q$, ending the entire path, or is between two points of $\neg P$, in which case we have a subpath of length 1. Otherwise, it is a path in which links from $R \cup S$ alternate with links from $1_{M}$, and that starts and ends with a link from $R \cup S$, and hence is of odd length. The subpath therefore gives an element of $\operatorname{Cut}(M ; R, S)$, and hence the entire path is in $\operatorname{Cut}(P ; \operatorname{Cut}(M ; R, S), T)$.

The fact that $1_{M}$ functions as a two-sided identity follows immediately from Lemma 7.

Finally, composition preserves inclusion of relations, and hence the category is order-enriched.

The two monoidal structures we need have products $\otimes$ and $\oplus$ and units $T$ and $\perp$. The associativity, identity and symmetry transformations are all given by relators which simply relate the corresponding pairs of literals. All of these transformations satisfy the conditions of Lemma 7 and from this the necessary naturality and coherence follow immediately.

Similarly the distributivity $\delta: A \otimes(B \oplus C) \longrightarrow(A \otimes B) \oplus C$ is also given by the relator relating corresponding literals, and hence is also natural and to satisfies the required coherence.

We have thus established that $\mathcal{C P}$ is a linearly distributive category.

Lemma 9. $\mathcal{C P}$ is a linearly distributive category with negation.

Proof. We established above that $\mathcal{C P}$ was a linearly distributive category. It remains to show the negation. On objects this is negation of matrices. We need maps $\neg M \otimes M \longrightarrow \perp$ and $\top \longrightarrow M \oplus \neg M$. We thus need relators for $M \oplus \neg M \oplus \perp$ and $\perp \oplus M \oplus \neg M$. These are essentially the identity on $M$. It is now easy to see that they satisfy the required coherence.

Moving on, we must show that the symmetric monoidal category $(\mathcal{C P}, \oplus, \perp)$ has symmetric monoids, and that $(\mathcal{C P}, \otimes, \top)$ has symmetric comonoids. For the first of these, we need identities given by relators [ $]_{M}$ on $\top \oplus M$ and multiplication given by relators $\nabla_{M}$ on $\neg M \oplus \neg M \oplus M$. These are the empty relator for [ [ $]_{M}$, and the obvious generalization of the identity for $\nabla_{M}$. It is easy to see that these satisfy the associativity and identity equations. The existence of comonoids is dual. 
Lemma 10. The object-indexed families of maps $\Delta_{M}, \nabla_{M},\langle\rangle_{M}$, and []$_{M}$ are lax natural transformations.

This is the point at which we start to need the order-enrichment, and the reason we have chosen ordering to be co-inclusion becomes clear.

Proof. Suppose $R \vdash \neg M \oplus N$, then \langle\rangle$_{M}$ is empty, while \langle\rangle$_{N} \circ R$ is $R \cap M \times M$, the set of elements of $R$ which are pairs of literals from $M$. It follows that \langle\rangle is a lax natural transformation. Turning now to $\Delta$, consider first the composite $(R \otimes R) \circ \Delta: \neg M \oplus(N \otimes N)$. We shall need to distinguish between the copies of $N$, and so use the convention that a subscript 0 indicates the first copy and a subscript 1 the second. We can calculate this composite using lemma 7 , or rather its slight generalization. Using this we see that the composite is the relator that includes the restriction of $R$ to $\neg M \times \neg M$, copies of the restriction of $R$ to $\neg M \times N$ on $\neg M \times N_{0}$ and $\neg M \times N_{1}$, and copies of the restriction of $R$ to $N \times N$ on $N_{0} \times N_{0}$ and $N_{1} \times N_{1}$. The alternative composite, $\Delta \circ R$, contains all of this, but also the copies of the restriction of $R$ to $N_{0} \times N_{1} . \Delta$ is hence lax natural.

The proofs of lax naturality for [] and $\nabla$ are identical.

Lemma 11. The object-indexed families of maps $\Delta_{M}, \nabla_{M},\langle\rangle_{M}$, and []$_{M}$ satisfy the inequalities $\Delta \nabla,\langle\rangle[], \nabla \Delta$, and []\langle\rangle .

Proof. It is easily seen that the inequalities $\Delta \nabla$ and $\nabla \Delta$ are actually equalities, while for \langle\rangle[] (and []\langle\rangle ) the composite round three sides of the square is the empty relator, and hence the inequality holds.

This completes:

Proposition 3. $\mathcal{C P}$ is a classical category.

Hence, the simple combinatorial structure of matrices and relators forms a properly structured model of classical proof theory. The only information we have in this is the link between literals induced from axioms. Nevertheless, the existence of the category tells us we still have a non-trivial model, carrying interpretations of the proof rules, and with subset on relators giving us an abstract interpretation of cut elimination.

\section{A final note}

We have chosen a definition of relator that allows it to include links that do not lie on any path, and hence cannot arise from a cut-free proof. The reason for doing so is that such links can be introduced by our definition of Cut. Nevertheless it is possible to construct a model in which we simply rule such links out. Note that the smallest substructure containing a link is either a horizontal or a vertical composite, and we can use this to describe links as horizontal or vertical. A horizontal link will lie on some path, and a vertical link on none. If we restrict relators to contain only horizontal links, then the only operation we use in our 
model that does not preserve this is Cut. However, we can simply restrict the result of Cut to be the subset of horizontal links. This will still be a relator, and it is easy to see that associativity and the other properties of composition are preserved. This gives us a second model, technically a reflective identity on objects subcategory of the first.

\section{References}

1. S. Abramsky and R. Jagadeesan. New foundations for the geometry of interaction. J. Pure Applied Algebra, 111(1):53-119, 1994.

2. P.B. Andrews. Theorem-proving via general matings. J.ACM, 28(2):193-214, 1981.

3. G. Bellin, J.M.E. Hyland, E.P. Robinson, and C. Urban. Categorical proof theory of classical propositional calculus. Theoretical Computer Science 364(2): 146-165, 2006.

4. W. Bibel. On matrices with connections. J.ACM, 28(4):633-645, 1981.

5. J.R.B. Cockett and R.A.G. Seely. Weakly Distributive Categories. J. Pure Appl. Algebra 114(2), 133-173, 1997.

6. K. Došen. Cut Elimination in Categories, Kluwer Academic Publishers, 1999.

7. C. Führmann and D. Pym. Order-enriched categorical models of the classical sequent calculus. J. Pure Appl. Algebra 204(1), 21-78, 2006.

8. C. Führmann and D. Pym. On the Geometry of Interaction for Classical Logic. In LICS 2004, 211-220, Turku (Finland), IEEE, 2004.

9. C. Führmann and D. Pym. On categorical models of classical logic and the geometry of interaction. Math. Struct. Comp. Sci. (2007) 17, 957-1027.

10. J.-Y. Girard, Y. Lafont, and P. Taylor. Proofs and Types. CUP, 1989.

11. Dominic J.D. Hughes. Proofs Without Syntax. Annals of Mathematics, 143(3), 1065-1076, 2006.

12. J.M.E. Hyland. Proof theory in the abstract. Ann. of Pure Appl. Logic, 114(13):43-78, 2002.

13. J.M.E. Hyland. Abstract interpretation of proofs: Classical propositional calculus. In Proceedings CSL 2004, LNCS 3210, 6-21, 2004.

14. F. Lamarche and L. Straßburger. Naming proofs in propositional classical logic. In Proc. TLCA 05, 2005.

15. F. Lamarche and L. Straßburger. Constructing Free Boolean Categories. In Proc. LICS 05. IEEE, 2005.

16. J. Lambek and P.J. Scott. Introduction to higher order categorical logic, volume 7 of Cambridge studies in advanced mathematics. Cambridge University Press, 1986.

17. R. McKinley. Classical Doctrines. Ph.D. thesis, University of Bath, 2006.

18. M. Parigot. $\lambda \mu$-calculus: an algorithmic interpretation of classical natural deduction. In Proc. LPAR'92, LNCS 624: 190-201, 1992.

19. D. Pym and E. Ritter. On the semantics of classical disjunction. J. Pure Applied Algebra, 159:315-338, 2001.

20. E.P. Robinson. Proof Nets for Classical Logic. J. Logic Computat., 13(5):777-797, 2003.

21. L.A. Wallen. Automated Deduction in Nonclassical Logics. MIT Press, 1990. 